\title{
Linear Matrix Inequalities in Analysis with Multipliers ${ }^{1}$
}

\author{
V. Balakrishnan ${ }^{2}$, Y. Huang ${ }^{3}$, A. Packard ${ }^{4}$ and J. Doyle ${ }^{3}$
}

\begin{abstract}
We show that a number of standard robustness tests can be reinterpreted as special cases of the application of the passivity theorem with the appropriate choice of multipliers. We show how these tests can be performed using convex optimization over linear matrix inequalities.
\end{abstract}

\section{Introduction}

A large class of robust control problems are posed in the setting shown in Figure 1.

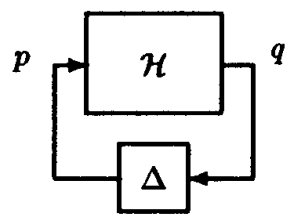

Figure 1: System $\mathcal{C}$ : A standard framework for robustness analysis

$\mathcal{H}$ is a stable linear time-invariant (LTI) system with transfer matrix $H(s)$. The perturbation $\Delta$, which in general is a nonlinear operator, appears in the feedback loop; it might represent nonlinearities and dynamics that are either unknown, unmodeled or neglected. (There are no external inputs or outputs, since we will be concerned only with the problem of stability, in the absence of inputs.) We will refer to the system shown in Figure 1 as $\mathcal{L}$. Often information about the "size" of $\Delta$ is available; most commonly, the $\mathbf{L}_{2}$ gain $^{5}$ of $\Delta$ is known or assumed to be less than or equal to some positive $\gamma$. In this case, the small gain theorem states that the system $\mathcal{L}$ is stable for all $\Delta$ (i.e., robustly stable) if $\|H\|_{\infty}<1 / \gamma$. Thus, the stability margin of the system, defined as the largest size of the perturbations against which the system is robustly stable, is at least $1 /\|H\|_{\infty}$.

\footnotetext{
${ }^{1}$ Research supported in part by the Control and Dynamical Systems group, Division of Engineering and Applied Sciences, California Institute of Technology, and by NSF under Grant NSFD CDR-88-03012.

${ }^{2}$ Institute for Systems Research, University of Maryland, College Park, Maryland. E-mail: raguesrc . und .edu.

${ }^{3}$ California Institute of Technology, Pasadena, California.

University of California, Berkeley, California.

${ }^{5}$ The notation and terminology are standard; see for example, [1].
}

Often, additional information is known or assumed about $\Delta: \Delta$ is diagonal or block-diagonal (i.e., "structured"); $\Delta$ is a convolution operator (i.e., "LTI perturbation"); $\Delta$ is a constant matrix (i.e., "parametric") etc. In these cases, a host of sufficient conditions for robust stability are available, each derived using apparently very different techniques. The first objective of this paper is to show that several of these sufficient conditions for robust stability can be derived in a single framework, that of the passivity theorem with multipliers. Other researchers who have made similar observations are Safonov and Chiang [2] and Hall and How [3]. The second objective of this paper is to show how these tests can be performed using convex optimization over linear matrix inequalities using state-space techniques.

The organization of the paper is as follows. In $\S 2$, we consider various robustness tests for system $\mathcal{L}$, and show how they can be rederived using the passivity theorem with multipliers. In $\S 3$, we show how these tests can be reduced to convex feasibility problems, in particular, feasibility of linear matrix inequalities. In $§ 4$, we present a simple numerical example.

\section{Robustness tests using the passivity theorem with multipliers}

We will first transform system $\mathcal{L}$ to the framework of the passivity theorem. To this end, we will apply a simple linear fractional transformation to the system, by defining new variables

$$
\tilde{p}=\gamma q-p, \quad \tilde{q}=\gamma q+p .
$$

This transformation is well-known in the literature; see for example [1]. It is referred to as the "bilinear sector transformation" in [2].

After routine algebraic manipulations, system $\mathcal{L}$ can be rewritten as system $\tilde{\mathcal{L}}$ shown in Figure 2, where $\mathcal{G}$ is a stable LTI system with transfer matrix $G(s)$ given by $G(s)=(I-\gamma H(s))^{-1}(I+\gamma H(s))$, and $\tilde{\Delta}=(\gamma I+\Delta)^{-1}(\gamma I-\Delta)$. It is readily checked that $\Delta$ has an $\mathbf{L}_{2}$ gain less than or equal to $\gamma$ if and only if $\tilde{\Delta}$ is passive, i.e., satisfies, for some real $\beta$,

$$
\int_{0}^{T} u(t)^{T}(\tilde{\Delta} u)(t) d t \geq \beta \text { for all } T \geq 0 \text { and all } u \text {. }
$$




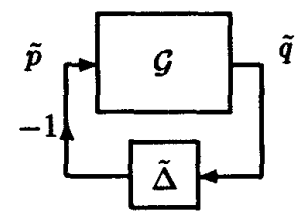

Figure 2: System $\tilde{\mathcal{L}}$ : Passivity theorem framework

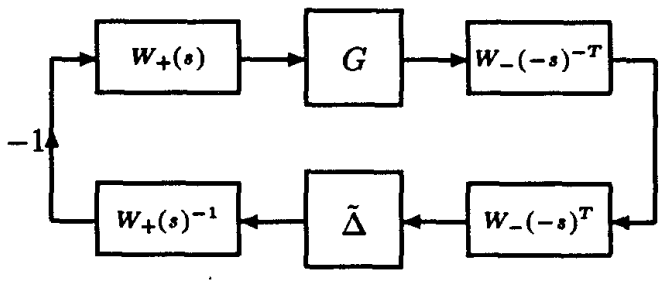

Figure 3: System with multipliers

We now apply the passivity theorem to system $\tilde{\mathcal{L}}$ : System $\tilde{\mathcal{L}}$ is robustly stable if $\mathcal{G}$ is strictly passive, which is equivalent (see [1], for example) to the frequency-domain condition that for some $\epsilon>0$,

$$
\lambda_{\min }\left(G(j \omega)+G(j \omega)^{*}\right) \geq 2 \epsilon I \text { for all } \omega \in \mathbf{R} .
$$

Suppose that in addition to being passive, $\tilde{\Delta}$ also satisfies additional assumptions. We can then employ multiplier theory to utilize this additional information. Consider the system in Figure 3, where $W_{+}(s)$ and $W_{-}(s)$ are transfer matrices of appropriate sizes. Suppose $W_{+}(s)$ and $W_{-}(s)$ satisfy

$W_{+}(s)$ and $W_{-}(-s)$ are stable and proper, with stable and proper inverses.

Then, robust stability of system in Figure 3 is equivalent to robust stability of the system $\tilde{\mathcal{L}}$.

Next, suppose that the operator

$$
W_{+}(s)^{-1} \circ \tilde{\Delta} \circ W_{-}(-s)^{T} \text { is passive. }
$$

(The symbol "o" denotes composition.) Then from the passivity theorem system, $\tilde{\mathcal{L}}$ is stable if

$$
W_{-}(-s)^{-T} G(s) W_{+}(s) \text { is strictly passive. }
$$

Condition (3) is equivalent to the frequency domain criterion that for some $\epsilon>0$,

$$
\begin{aligned}
& W_{-}(j \omega)^{-*} G(j \omega) W_{+}(j \omega) \\
& +W_{+}(j \omega)^{*} G(j \omega)^{*} W_{-}(j \omega)^{-1} \geq 2 \epsilon I,
\end{aligned}
$$

which in turn is equivalent to the condition that for some $\epsilon$,

$G(j \omega) W(j \omega)+W(j \omega)^{*} G(j \omega)^{*} \geq 2 \epsilon I$ for all $\omega \in \mathbf{R}$, where $W=W_{+} W_{-}$is called the stability multiplier.

Thus, robust stability analysis using multipliers involves finding $W$ such that

1. $W$ can be factorized into $W_{+} W_{-}$where $W_{-}$and $W_{+}$satisfy (1) and (2).

2. $W$ and $G$ satisfy (4).

The additional information about $\Delta$, and therefore $\tilde{\Delta}$, influences the choice of $W=W_{+} W_{-}$through condition (2).

With the substitution

$$
G(s)=(I-\gamma H(s))^{-1}(I+\gamma H(s)),
$$

condition (4) is equivalent to the condition that for some $\epsilon>0$,

$$
\begin{aligned}
& W_{\mathbf{H}}(j \omega)-\gamma^{2} H(j \omega) W_{\mathbf{H}}(j \omega) H(j \omega)^{*} \\
& +\gamma\left(H(j \omega) W_{\mathrm{S}}(j \omega)-W_{\mathrm{S}}(j \omega) H(j \omega)^{*}\right) \geq 2 \epsilon I \\
& \quad \text { for all } \omega \in \mathbf{R}
\end{aligned}
$$

where $W_{\mathrm{H}}(s)=W(s)+W(-s)^{T}$ and $W_{\mathrm{S}}(s)=W(s)-$ $W(-s)^{T}$.

With these preliminaries, we now consider how some standard robustness tests for system $\mathcal{L}$ can be performed for system $\tilde{\mathcal{L}}$.

\subsection{Diagonal perturbations}

Suppose that $\Delta$ in addition to having an $\mathbf{L}_{2}$ gain less than $\gamma$, is also diagonal. By this, we mean that the operator $\Delta$ can be also specified by $p_{i}=\delta_{i} q_{i}$, where each single-input single-ouput nonlinear operator $\delta_{i}$ has an $\mathbf{L}_{2}$ gain less than $\gamma$. (The extension to the blockdiagonal case, or when there are additional equality constraints such as $\delta_{i}=\delta_{j}$ is straightforward.) Note that $\tilde{\Delta}$ is diagonal as well.

Let $W$ be any constant diagonal positive-definite matrix. Then $W$ can be factored into $W_{+} W_{-}$with $W_{+}>0$ and $W_{-}>0$, which satisfy (1) and (2) (for instance, we can take $W_{+}=W, W_{-}=I$ ). Therefore, system $\mathcal{L}$ is robustly stable if for some diagonal $W>0$, and some $\epsilon>0$

$$
G(j \omega) W+W G(j \omega)^{*} \geq 2 \epsilon I \text { for all } \omega \in \mathbf{R} .
$$

Correspondingly, condition (5) is that for some $\epsilon>0$,

$$
W-\gamma^{2} H(j \omega) W H(j \omega)^{*} \geq 2 \epsilon I \text { for all } \omega \in \mathbf{R} .
$$

Inequality (7) is the well-known condition that the scaled $\mathbf{H}_{\infty}$ norm of $H$ be less than $1 / \gamma$.

\subsection{Diagonal LTI perturbations}

Next, suppose that $\Delta$ is a diagonal LTI perturbation with $\mathbf{L}_{2}$ gain less than or equal to $\gamma$. Then $\tilde{\Delta}$ is diagonal and passive. 
Suppose $W(s)$ is diagonal, bounded in the right-half complex plane and satisfies, for some $\epsilon>0$,

$$
W(j \omega)=W(j \omega)^{*} \geq 2 \epsilon I \text { for all } \omega \in \mathbf{R} .
$$

Then $W$ can be factorized into $W_{+}$and $W_{-}$satisfying (1) (see $[1, \S 9.4-5]$. Moreover, the factors $W_{-}$ and $W_{+}$thus obtained satisfy condition (2). This can be seen as follows. Since $\tilde{\Delta}$ is an LTI perturbation, condition (2) is equivalent to the frequency-domain condition that

$$
\begin{aligned}
& W_{+}(j \omega)^{-1} \tilde{\Delta}(j \omega) W_{-}(j \omega)^{*} \\
& +W_{-}(j \omega) \tilde{\Delta}(j \omega)^{*} W_{+}(j \omega)^{-*} \geq 0
\end{aligned}
$$

for all $\omega \in \mathbf{R}$, or equivalently

$$
\begin{aligned}
& W_{-}(j \omega)^{-1} W_{+}(j \omega)^{-1} \tilde{\Delta}(j \omega) \\
& +\tilde{\Delta}(j \omega) W_{+}(j \omega)^{-*} W_{-}(j \omega)^{-*} \geq 0
\end{aligned}
$$

for all $\omega \in \mathbf{R}$. The condition that $\tilde{\Delta}$ is passive means that the Nyquist plot of each diagonal entry of $\tilde{\Delta}$ lies in the closed right half complex plane. Since the product $W=W_{+} W_{-}$satisfies, for some $\epsilon>0$, condition (8), the Nyquist plot of $W^{-1} \tilde{\Delta}$ also lies in the closed right half complex plane, i.e., condition (9) is satisfied.

Let us next examine (4). In this case, we require, for some $\epsilon>0$,

$$
G(j \omega) W(j \omega)+W(j \omega) G(j \omega)^{*} \geq 2 \epsilon I \text { for all } \omega \in \mathbf{R},
$$

where $W$ satisfies (8). Correspondingly, condition (5) is that for some $\epsilon>0$,

$W(j \omega)-\gamma^{2} H(j \omega) W(j \omega) H(j \omega)^{*} \geq 2 \epsilon I$ for all $\omega \in \mathbf{R}$.

Inequality (11) is the well-known condition that the $\mathbf{H}_{\infty}$ norm of $H$, minimized over all frequencydependent scalings be less than $1 / \gamma$.

\subsection{Diagonal parametric perturbations}

Next, suppose that $\Delta$ is a constant diagonal matrix with entries known to lie in $[-\gamma, \gamma]$. Then $\tilde{\Delta}$ is a constant diagonal matrix with entries in $[0, \infty)$.

Suppose $W(s)$ is diagonal, bounded in the right half complex plane, and satisfies, for some $\epsilon>0$,

$$
W(j \omega)+W(j \omega)^{*} \geq 2 \epsilon I \text { for all } \omega \in \mathbf{R} .
$$

Then $W$ can be factorized into $W_{+}$and $W_{-}$satisfying (1). Moreover, the factors $W_{-}$and $W_{+}$thus obtained satisfy condition (2). This can be seen as follows. Since $\tilde{\Delta}$ is a constant diagonal matrix with nonnegative diagonal entries, condition (2) is equivalent to the frequency-domain condition that

$$
\begin{aligned}
& W_{+}(j \omega)^{-1} \tilde{\Delta} W_{-}(j \omega)^{*} \\
& +W_{-}(j \omega) \tilde{\Delta} W_{+}(j \omega)^{-*} \geq 0
\end{aligned}
$$

for all $\omega \in \mathbf{R}$, or equivalently

$$
\begin{aligned}
& W_{-}(j \omega)^{-1} W_{+}(j \omega)^{-1} \tilde{\Delta} \\
& +\tilde{\Delta} W_{+}(j \omega)^{-*} W_{-}(j \omega)^{-*} \geq 0
\end{aligned}
$$

for all $\omega \in \mathbf{R}$. In other words, we require the Nyquist plot of every diagonal entry of $\tilde{\Delta} W^{-1}$ to lie in the closed right half plane. Since $\tilde{\Delta}$ is a constant diagonal positive semi-definite matrix, we simply require every diagonal entry of $W$ to have a Nyquist plot that lies in the closed right half plane; this is ensured by condition (12).

Let us next examine the condition (4). We then require, for some $\epsilon>0$,

$$
G(j \omega) W(j \omega)+W(j \omega)^{*} G(j \omega)^{*} \geq 2 \epsilon I .
$$

In this case inequality (5) is that for some $\epsilon>0$,

$$
\begin{gathered}
W_{\mathbf{H}}(j \omega)-\gamma^{2} H(j \omega) W_{\mathbf{H}}(j \omega) H(j \omega)^{*} \\
+\gamma\left(H(j \omega) W_{\mathbf{S}}(j \omega)-W_{\mathbf{S}}(j \omega) H(j \omega)^{*}\right) \\
\geq 2 \epsilon I \text { for all } \omega \in \mathbf{R},
\end{gathered}
$$

which is well-known condition condition due to Fan, Tits and Doyle [4] for robust stability with real parametric perturbations.

Remark. The robustness test for parametric perturbations also ensures robust stability when $\Delta$ is a diagonal LTI perturbation satisfying $\Delta(j \omega)=\Delta(j \omega)^{*}$ with $\Delta(j \omega)^{*} \Delta(j \omega) \leq \gamma^{2} I$.

\section{Numerical implementation of stability tests using LMIs}

We now show how the tests described in the previous section can be performed using convex optimization techniques.

The fundamental result that enables us to check the frequency domain conditions of the previous section is the following: A transfer matrix $\tilde{H}$ with state-space realization $(\tilde{A}, \tilde{B}, \tilde{C}, \tilde{D})$ satisfies

$$
\tilde{H}(j \omega)+\tilde{H}(j \omega)^{*} \geq 2 \epsilon I \text { for all } \omega \in \mathbf{R},
$$

if and only if there exists a symmetric matrix $Q$ satisfying the matrix inequality

$$
\left[\begin{array}{cc}
\tilde{A} Q+Q \tilde{A}^{T} & \tilde{B}-Q \tilde{C}^{T} \\
\tilde{B}^{T}-\tilde{C} Q & 2 \epsilon I-\left(\tilde{D}+\tilde{D}^{T}\right)
\end{array}\right] \leq 0
$$

(see for example, [5]). This matrix inequality is affine in the variable $Q$, and is referred to as a linear matrix inequality or LMI in $Q$. The important point here is that an LMI is a convex constraint with a special structure, and consequently, there exist efficient algorithms for checking its feasibility. We refer the reader to $[6,7]$ for details. 
Let $(A, B, C, D)$ be a state-space realization of $H(s)$. Then it is easy to verify that

$$
G(s)=(I-\gamma H(s))^{-1}(I+\gamma H(s))
$$

has a state space realization $\left(A_{G}, B_{G}, C_{G}, D_{G}\right)$, where

$$
\begin{aligned}
& A_{G}=A+\gamma B(I-\gamma D)^{-1} C, \\
& B_{G}=2 B(I-\gamma D)^{-1} \\
& C_{G}=(I-\gamma D)^{-1} \gamma C \\
& D_{G}=(I+\gamma D)(I-\gamma D)^{-1}
\end{aligned}
$$

\subsection{Diagonal perturbations}

$W>0$ is a constant diagonal. Then $G W$ has a statespace realization $\left(A_{G W}, B_{G W}, C_{G W}, D_{G W}\right)$ where

$$
\begin{array}{ll}
A_{G W}=A_{G}, & B_{G W}=B_{G} W, \\
C_{G W}=C_{G}, & D_{G W}=D_{G} W .
\end{array}
$$

Therefore, condition (6) is equivalent to the LMI in $Q=Q^{T}$, diagonal $W>0$ and $\epsilon>0$ :

$$
\left[\begin{array}{cc}
A_{G} Q+Q A_{G}^{T} & B_{G} W-Q C_{G}^{T} \\
W B_{G}^{T}-C_{G} Q & 2 \epsilon I-\left(D_{G} W+W D_{G}^{T}\right)
\end{array}\right] \leq 0 .
$$

\subsection{Diagonal LTI perturbations}

The problem here is to check if there exists $W$ satisfying (8) so that condition (10) is satisfied. In order to perform this test numerically, we restrict $W$ to lie in a subspace

$$
\mathcal{W} \triangleq\left\{W_{0}+\sum_{i=1}^{m} \theta_{i} W_{i} \mid \theta \in \mathbf{R}^{m}, \quad W_{i}(s)=W_{i}(-s)^{r}\right\},
$$

where $W_{i}$ are fixed transfer matrices. Thus, every $W \in \mathcal{W}$ has a realization $\left(A_{W}, B_{W}(\theta), C_{W}, D_{W}(\theta)\right.$ with $B_{W}$ and $D_{W}$ affine functions of $\theta$. Therefore, condition (8) is equivalent to an LMI in $Q_{W}=Q_{W}^{T}$, $\theta$ and $\epsilon>0$ :

$$
\left[\begin{array}{cc}
A_{W} Q_{W}+Q_{W} A_{W}^{T} & B_{W}(\theta)-Q_{W} C_{W}^{T} \\
B_{W}(\theta)^{T}-C_{W} Q_{W} & 2 c I-\left(D_{W}(\theta)+D_{W}(\theta)^{T}\right)
\end{array}\right] \leq 0 .
$$

Next, $G W$ has a state-space realization $\left(A_{G W}, B_{G W}\right.$, $\left.C_{G W}, D_{G W}\right)$ where

$$
\begin{aligned}
A_{G W} & =\left[\begin{array}{cc}
A_{W} & 0 \\
B_{G} C_{W} & A_{G}
\end{array}\right], B_{G W}(\theta)=\left[\begin{array}{c}
B_{W}(\theta) \\
B_{G} D_{W}(\theta)
\end{array}\right], \\
C_{G W} & =\left[\begin{array}{ll}
D_{G} C_{W} & C_{G}
\end{array}\right], D_{G W}(\theta)=D_{G} D_{W}(\theta) .
\end{aligned}
$$

Note that $B_{G W}$ and $D_{G W}$ are affine functions of $\theta$.

Then checking condition (10) is equivalent to the LMI in variables $Q=Q^{T}, \theta$ and $\epsilon>0$ :

$$
\left[\begin{array}{cc}
Q A_{G W}^{T}+A_{G W} Q & B_{G W}(\theta)-Q C_{G W}^{T} \\
B_{G W}(\theta)^{T}-C_{G W} Q & \left(\begin{array}{c}
2 \epsilon I-D_{G W}(\theta) \\
-D_{G W}(\theta)^{T}
\end{array}\right)
\end{array}\right] \leq 0 .
$$

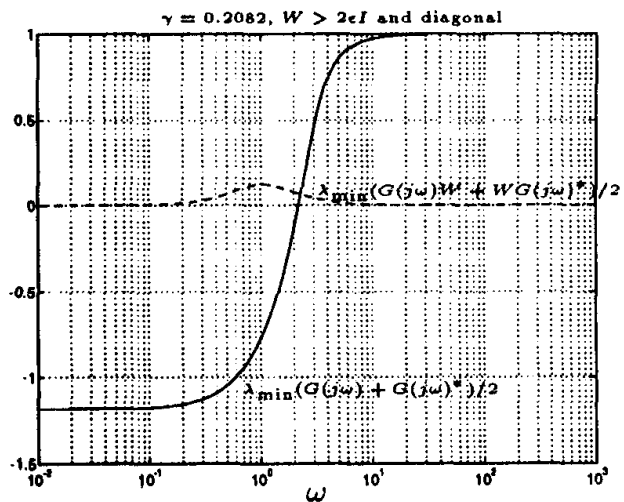

Figure 4: Diagonal perturbations

\subsection{Diagonal parametric perturbations}

The numerical procedure here is very similar to the one in the previous section. Here we restrict the multipliers $W$ to lie in a subspace

$$
\mathcal{W} \triangleq\left\{W_{0}+\sum_{i=1}^{m} \theta_{i} W_{i} \mid \theta \in \mathbf{R}^{m}\right\}
$$

where $W_{i}$ are fixed transfer matrices. Note that unlike with LTI perturbations, we do not require $W_{i}(s)=W_{i}(-s)^{T}$.

Once again, condition (12) is equivalent to the LMI condition (18), and checking condition (14) is equivalent to the LMI (19).

\section{An example}

We take

$$
H(s)=\left[\begin{array}{cc}
2 & \frac{-10 s-8}{5(s+1)} \\
\frac{-2 s+8}{(s+1)} & 2
\end{array}\right] .
$$

\subsection{Diagonal perturbations}

We first consider the case when $\Delta$ is diagonal. We find, using LMI (16) and a simple bisection scheme, that the largest size $\gamma$ of diagonal $\Delta$ against which the system is guaranteed to be stable (using our approach) is about 0.2082 ; the corresponding multiplier is $W=\operatorname{diag}(0.3867,1.3454)$. A plot of the minimum eigenvalues of the Hermitian parts of $G(j \omega)$ and $G(j \omega) W$ are shown in Figure 4 .

\subsection{Diagonal LTI perturbations}

We next consider diagonal LTI $\Delta$. The multipliers we employ are diagonal, with diagonal entries of the form $w_{0}+w_{1} /\left(s^{2}-1\right)$. Then, using LMIs (18) and (19) and a simple bisection scheme, that the largest size $\gamma$ of 


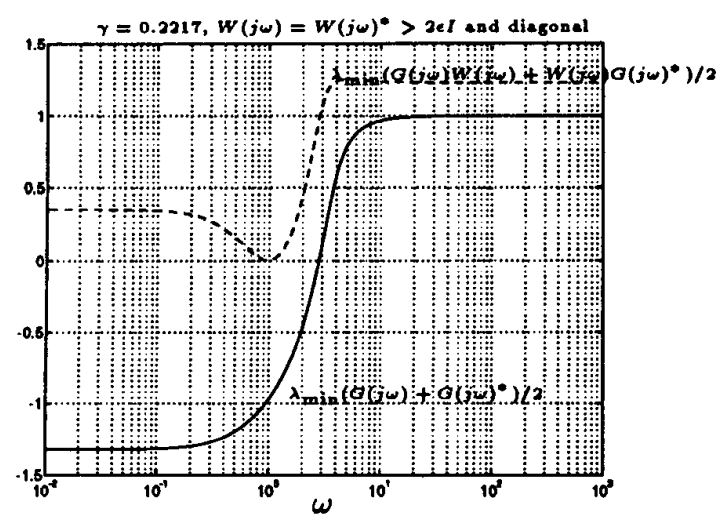

Figure 5: Diagonal LTI perturbations

diagonal LTI $\Delta$ against which the system is guaranteed to be stable is about 0.2217 ; the corresponding multiplier $W(s)$ is

$$
\begin{array}{r}
\operatorname{diag}\left(1.3299-0.8419 /\left(s^{2}-1\right)\right. \\
\left.1.1755-8.9447 /\left(s^{2}-1\right)\right) .
\end{array}
$$

A plot of the minimum eigenvalues of the Hermitian parts of $G(j \omega)$ and $G(j \omega) W(j \omega)$ are shown in Figure 5 .

\subsection{Diagonal parametric perturbations} When $\Delta$ is a constant, unknown diagonal matrix, we use diagonal multipliers $W(s)$ with diagonal entries of the form $w_{0}+w_{1} /(s+1)+w_{2} /(s+1)^{2}$. Then, using LMIs (18) and (19) and a simple bisection scheme, we find that the largest size $\gamma$ of diagonal parametric $\Delta$ against which the system is guaranteed to be stable is about 0.2414 ; the corresponding multiplier is

$$
\begin{gathered}
\operatorname{diag}\left(0.2961+0.1206 /(s+1)-0.0934 /(s+1)^{2},\right. \\
\left.0.5021+1.2464 /(s+1)-0.1630 /(s+1)^{2}\right) .
\end{gathered}
$$

A plot of the minimum eigenvalues of the Hermitian parts of $G(j \omega)$ and $G(j \omega) W(j \omega)$ are shown in Figure 6.

\section{Conclusions}

We have reinterpreted a number of standard robustness tests in the framework of the passivity theorem with multipliers. We have also shown how these tests can be performed using convex optimization over linear matrix inequalities. Several extensions to the results presented here are possible. The first is efficient computation of the largest $\gamma$ for which we can guarantee robust stability using multipliers (recall that we have used a bisection scheme in this paper). This quantity has the interpretation of a lower bound on

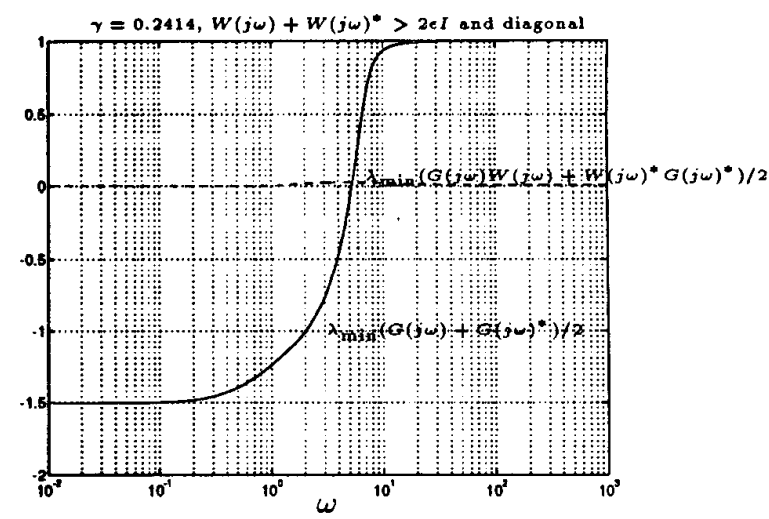

Figure 6: Diagonal parametric perturbations

the robust stability margin [2] (and yields an upper bound on " $\mu$ " [8]). The second is feedback synthesis (or robust stabilization). Finally, the extension of the techniques presented in this paper to the computation of robust performance measures is also of interest.

\section{References}

[1] C. A. Desoer and M. Vidyasagar. Feedback Systems: Input-Output Properties. Academic Press, New York, 1975.

[2] M. G. Safonov and R. Y. Chiang. Real/complex $K_{m}$-synthesis without curve fitting. In C. T. Leondes, editor, Control and Dynamic Systems, volume 56, pages 303-324. Academic Press, New York, 1993.

[3] S. R. Hall and J. P. How. Mixed $\mathbf{H}_{2} / \mu$ performance bounds using dissipation theory. In Proc. IEEE Conf. on Decision and Control, San Antonio, Texas, December 1993.

[4] M. K. H. Fan, A. L. Tits, and J. C. Doyle. Robustness in the presence of mixed parametric uncertainty and unmodeled dynamics. IEEE Trans. Aut. Control, AC-36(1):25-38, January 1991.

[5] B. D. O. Anderson and S. Vongpanitlerd. Network analysis and synthesis: a modern systems theory approach. Prentice-Hall, 1973.

[6] Yu. Nesterov and A. Nemirovsky. Interiorpoint polynomial methods in convex programming, volume 13 of Studies in Applied Mathematics. SIAM, Philadelphia, PA, 1994.

[7] S. Boyd, L. El Ghaoui, E. Feron, and V. Balakrishnan. Linear matrix inequalities in systems and control theory, 1994. To be published by SIAM.

[8] J. Doyle. Analysis of feedback systems with structured uncertainties. IEE Proc., 129-D(6):242250, November 1982. 